\title{
Proposed Measurement of an Effective Flux Quantum in the Fractional Quantum Hall Effect
}

\author{
J.K. Jain ${ }^{1}$, S.A. Kivelson ${ }^{2}$, and D.J. Thouless ${ }^{3}$ \\ 1. Department of Physics, State University of New York at Stony Brook, Stony Brook, New \\ York, 11794-3800 \\ 2. Department of Physics, University of California at Los Angeles, Los Angeles, California \\ 90024 \\ 3. Department of Physics, University of Washington, Seattle, Washington, 98195
}

\begin{abstract}
We consider a channel of an incompressible fractional-quantum-Hall-effect (FQHE) liquid containing an island of another FQHE liquid. It is predicted that the resistance of this channel will be periodic in the flux through the island, with the period equal to an odd integer multiple of the fundamental flux quantum, $\phi_{0}=h c / e$. The multiplicity depends on the quasiparticle charges of the two FQHE liquids.
\end{abstract}


Since the seminal works of Laughlin [1] and Halperin [2], it has been recognized that the elementary excitations (quasiparticles) in the fractional quantum Hall effect (FQHE) [3] have fractional charge and obey fractional statistics. These fractional quantum numbers essentially follow from the incompressibility at fractional filling factors, and their values can be determined from rather general principles [4]. For the principal FQHE liquids at filling factors [5]

$$
\nu_{n} \equiv \frac{n}{2 n+1}
$$

the charge of a quasihole is

$$
e_{n}=\frac{e}{2 n+1}
$$

while its statistics is

$$
\theta_{n}=\frac{2 n-1}{2 n+1}
$$

defined so that an exchange of two quasiholes produces a phase factor of $e^{i \pi \theta}$ [6]. It has been argued that the fractionally quantized Hall resistance itself is a measurement of the charge of the quasiparticles [7], but, on the other hand, the Hall resistance is a property of the condensate and therefore does not directly probe the excitations [8]. The observation of the 'hierarchy fractions' has been cited as evidence for the fractional statistics of quasiparticles [9], but it is clear that all fractions can be understood without reference to quasiparticles at all [10]. Several experiments have reported evidence for the fractional charge [11]. However, their theoretical interpretation is either not unique, or not completely understood. A definitive and direct observation of the fractional charge or the fractional statistics of the quasiparticles is therefore lacking.

In order to illustrate the basic conceptual difficulty with the measurement of the fractional charge, consider the Aharonov-Bohm (AB) geometry in Fig.1a. In the FQHE regime, the current is carried by fractionally charged quasiparticles, so it is tempting to expect that the properties of the system, such as the resistance, will be periodic in the flux with period $\phi_{0}^{*}=h c / e_{n}$, in analogy with the argument of Byers and Yang (BY) [12. However, in any true $\mathrm{AB}$ geometry, the period must always be $\phi_{0}=h c / e$. The reason is that while the quasiparticles may provide an effective description, the fundamental particles are still electrons [13]. In fact, periods greater than $\phi_{0}$ are ruled out by the BY argument (while 
smaller periods are, of course, possible and do occur, e.g. in the case of superconductors).

In this Letter, we consider a resonant tunneling experiment and predict that, under certain conditions, the resistance will exhibit approximate periodicity in flux with period equal to an odd integer multiple of $\phi_{0}$. An observation of this periodicity should provide direct and unambiguous evidence of the existence of fractional quantum numbers in the FQHE. There have been other proposals for the observation of the fractional quantum numbers [14], but they deal with non-equilibrium situations. The experiment proposed in the present work, on the other hand, probes an equilibrium property of the system.

We consider the geometry of Fig.1b, in which a (narrow) channel of $\nu^{\prime}=p^{\prime} / q^{\prime} \mathrm{FQHE}$ liquid (where $p^{\prime}$ and $q^{\prime}$ are relatively prime integers) contains an island of area $A$ of the $\nu=p / q$ FQHE liquid. This could be produced experimentally by creating a gentle potential hill or valley with the help of an external gate. The chemical potential at the edges of the sample is assumed to be fixed externally. The BY argument clearly does not apply in this situation, since electrons occupy the entire sample. It is possible for a quasiparticle to tunnel from one edge of the channel to the other, which is actually a tunneling between two many-body configurations, one in which the quasiparticle is on one edge, and the other in which it is on the other. The tunneling amplitude determines the longitudinal resistance, as was shown in a Landauer-type formulation of the QHE [15]. The longitudinal resistance exhibits peaks whenever there is resonant tunneling from one edge of the sample to the other through a quasi-bound state on the potential island [16]. The main conclusion of this work is that successive peaks occur when the flux through the island changes by

$$
j \phi_{0}=\frac{q}{s} \phi_{0}
$$

where $s$ is an integer, equal to the highest common factor of $q$ and $q^{\prime}$. Since $q$ and $q^{\prime}$ are, in general, odd integers, $j$ is also an odd integer. Note that $j$ depends only on $q$ and $q^{\prime}$, i.e., only on the quasiparticle charges of the two FQHE liquids.

To give the simplest derivation of this result, let us change the flux through the $\nu=p / q$ island liquid in a way that no quasiparticles (quasiholes or quasielectrons) are created in the bulk. This can be achieved by spreading the additional flux over a sufficiently large area of the island. The additional flux $j \phi_{0}$ contracts the island liquid, so that an additional charge 
$j e p / q$ is required to restore the edge of the island FQHE liquid to its original state. Since the charge must be supplied by $j^{\prime}$ quasiparticles of the channel $\left(\nu^{\prime}\right)$ FQHE liquid, we must have

$$
j \frac{p}{q}=j^{\prime} \frac{1}{q^{\prime}}
$$

which leads to the period $j \phi_{0}$ given by Eq. (4). In particular, if $\nu=0$, i.e. if the island is charge free, the period is $\phi_{0}$, since the channel FQHE liquid can return to its original state by the transfer of $p^{\prime}$ quasiparticles from the outer edge to the inner edge. (Thus, $\nu=0$ is to be interpreted as $\nu=0 / 1$ for the purpose of Eq. 4 .) This is equivalent to a gauge transformation of the original wave function.

Let us now give a more microscopic description, which takes account of the internal structure of the various FQHE liquids. We use the framework of the composite fermion (CF) theory [10], in which the the wave function of the $\nu_{n}$ FQHE liquid is given by

$$
\chi_{n /(2 n+1)}=\prod_{j<k}\left(z_{j}-z_{k}\right)^{2} \Psi_{n},
$$

where $\Psi_{n}$ is the wave function of $n$ filled Landau levels (LLs), and $z_{j}=x_{j}+i y_{j}$ denotes the position of the $j$ th electron. Consider the situation when the island FQHE liquid is $\nu_{n-1}$ and the channel FQHE liquid is $\nu_{n}$. This state corresponds to an integer quantum Hall effect (IQHE) state which has $n$ filled LLs everywhere except in an island where the filling factor is $n-1$. An integer number $(K)$ of electrons have been removed from the $n$th LL to create the island [17]. In the IQHE state $\Psi$, each hole has an excess charge $e$ associated with it. Upon multiplication by the Jastrow factor, $\prod_{j<k}\left(z_{j}-z_{k}\right)^{2}$, which converts each electron into a CF, each hole in the $n$th LL of $\Psi$ becomes a quasihole of the $\nu_{n}$ liquid, with an excess charge $e_{n}=e /(2 n+1)$ associated with it [18]. Therefore, for $K$ quasiholes, there is a net deficiency of charge $K e_{n}$ in the island region. This deficiency is related to the difference between the filling factors outside and inside the island as:

$$
K e_{n}=\left(\nu_{n}-\nu_{n-1}\right)\left(\Phi / \phi_{0}\right)
$$

where $\Phi=A B$ is the flux through the island. Thus, for $K$ quasiholes, the flux through the island is given by

$$
\Phi=K(2 n-1) \phi_{0} .
$$


Addition or removal of a single quasihole requires a flux change of $(2 n-1) \phi_{0}$ through the island, which gives the period

$$
\Delta \Phi=(2 n-1) \phi_{0}
$$

When the island liquid is $\nu_{n+1}$ (and the channel liquid is $\nu_{n}$ ),

$$
K e_{n}=\left(\nu_{n+1}-\nu_{n}\right)\left(\Phi / \phi_{0}\right),
$$

and the period is given by

$$
\Delta \Phi=(2 n+3) \phi_{0}
$$

In both cases, the periods are in agreement with the general formula, Eq. (位).

It is instructive to consider this problem from yet another perspective. We write pseudo wave functions in terms of the coordinates of the quasiparticles, treating them as point particles [2]. First consider the situation when the channel liquid is $\nu_{n}$ and the island liquid is $\nu_{n-1}$. Since the low-energy states contain quasiholes in the topmost level only (i.e., related to holes only in the $n$th LL of $\Psi_{n}$ ), they fill a lowest LL of their own. The most energetically favorable situation is when they completely fill the LL. The wave function is then

$$
\prod_{j<k}\left(\eta_{j}-\eta_{k}\right)^{\theta} \exp \left[-\frac{1}{4} \sum_{j=1}^{K} \frac{\left|\eta_{j}\right|^{2}}{l_{n}^{2}}\right],
$$

where $\eta_{j}$ denote the positions of the quasiholes, and $l_{n}^{2}=\hbar c / e_{n} B$. The area of the island is given by (neglecting irrelevant corrections of order unity) [14a]

$$
A=K \frac{\phi_{0}}{B} \frac{\theta}{\left(e_{n} / e\right)}
$$

With $\theta=\theta_{n}$, given by Eq. (3), this is identical to Eq. (8), and gives a period of $(2 n-1) \phi_{0}$. In the other case, when the island liquid is $\nu_{n+1}$, we write the quasielectron wave function [2, 19]

$$
\prod_{j<k}\left(\bar{\eta}_{j}-\bar{\eta}_{k}\right)^{-\theta} \exp \left[-\frac{1}{4} \sum_{j=1}^{K} \frac{\left|\eta_{j}\right|^{2}}{l_{n}^{2}}\right],
$$

where now $\eta_{j}$ are the quasielectron coordinates. In this case, one is tempted to choose the quasiparticle statistics $\theta=\theta_{n}$. However, in order for the quasielectron wave function to be regular as two quasielectrons approach one another, which is required by the hermiticity of the Hamiltonian [20], we must choose the statistics to be [21]

$$
\theta=\theta_{n}-2=-\frac{2 n+3}{2 n+1} .
$$


(The resulting quasielectron wave function can also be interpreted as a FQHE liquid of quasielectrons of statistics $\theta_{n}$ [2].) The period from Eq. (13) is $(2 n+3) \phi_{0}$, as expected. From this perspective, the period can be interpreted as a measure of the ratio of the statistics to charge of the quasiparticles of the channel FQHE liquid (see Eq. 13).

We close with the following remarks.

(i) The above arguments actually show that for a consistent description in terms of quasiparticles, they must be assigned fractional statistics. Similar arguments had originally led Halperin to discover that quasiparticles obey fractional statistics [2].

(ii) It is interesting to see how the BY result is obtained from the perspective of the quasiparticles. This pertains to the situation when charge is completely depleted from the island region. In the CF theory, this relates to the IQHE state in which all $n$ LLs are empty in the island region. In the quasihole language, $n$ LLs of quasiholes are occupied. In analogy with the CF theory, the wave function of this quasihole state is given by [21]

$$
\prod_{j<k}\left(\eta_{j}-\eta_{k}\right)^{\theta_{n}-1} \Psi_{n}
$$

The size of the droplet described by this wave function is such that the flux through it is given by

$$
\Phi=\frac{K}{n} \frac{\phi_{0}}{B}
$$

In this case, the number of quasiholes increases in units of $n$ (since, whenever it is possible to add a quasihole in one level, it is possible in other levels as well), and we recover the BY period of $\phi_{0}$.

(iii) We have so far assumed that the $\nu=p / q$ FQHE liquid in the island is ideal. It is easy to see that the presence of a fixed number of quasielectrons or quasiholes in this liquid will not alter the period. Whenever a new quasiparticle is created, the periodic sequence will suffer a phase shift. The same will be true when there are lakes of other FQHE liquids inside the island; the period will remain $j \phi_{0}$ except when a new quasiparticle is created in one of the lakes. Thus, in general, we expect finite sequences of peaks in the longitudinal resistance with the predicted spacing. The larger the amount of the $\nu=p / q$ fluid in the island, the longer will be the length of the sequence. 
(iv) We have neglected the Coulomb blockade effects [22], which are expected to be small for sufficiently large islands. These are also well understood and may be subtracted out to reveal the effects discussed here. We note that the periodicity of the effect does not depend on the structure of the interface between the two FQHE liquids, so long as it is narrow compared to the regions of the FQHE liquids.

(v) Any $j \phi_{0}$ periodicity $(j \neq 1)$ in the situation when the island is completely depleted, as is presumably the case in the experiment of Simmons et al. [11], must be a non-equilibrium effect [14]. This should be experimentally testable.

In conclusion, we predict conditions under which an interference between two FQHE liquids allows the observation of an effective flux quantum, which is equal to an odd integer multiple of the fundamental flux quantum. The period depends on the quasiparticle charges of the two FQHE liquids; in the case of two successive FQHE states of a sequence, it can be also interpreted as a measure of the ratio of the statistics to the charge of the quasiparticles of the channel FQHE liquid. This experiment should also serve as a probe into the internal structure of the FQHE liquids.

We thank V.J. Goldman for discussions and comments. This work was supported in part by the National Science Foundation under Grants Nos. DMR90-20637, DMR90-11803, and DMR92-20733. We acknowledge the hospitality of the Aspen Center for Physics. 


\section{References}

[1] R.B. Laughlin, Phys. Rev. Lett. 50, 1395 (1983).

[2] B.I. Halperin, Phys. Rev. Lett. 52, 1583 (1984).

[3] D.C. Tsui, H.L. Störmer, and A.C. Gossard, Phys. Rev. Lett. 48, 1559 (1982).

[4] W.P. Su, Phys. Rev. B 34, 1031 (1986). This work shows that the quasiparticle charge and statistics (mod 2) can be determined completely by assuming that there is only one type of quasiparticle, and its charge assumes the largest allowed value.

[5] The filling factor at magnetic field $B$ is defined by $\nu=\rho \phi_{0} / B$ where $\rho$ is the electron density, and $\phi_{0}=h c / e$.

[6] In general, the charge of the quasihole of the $\nu=p / q$ liquid is given by $e / q$ and the statistics is given by $\theta=p^{\prime} / q$ where $p^{\prime} p=1 \bmod q$. This simple expression for the statistics (which is, of course, in agreement with those of Refs. [2, 国) was given by G. Moore and N. Read, Nucl. Phys. B 360, 362 (1991).

[7] R.B. Laughlin in The Quantum Hall Effect, Eds. R.E. Prange and S.M. Girvin, 2nd edition (Springer-Verlag, NY).

[8] S.A. Kivelson and V.L. Pokrovsky, Phys. Rev. B 40, 1373 (1989).

[9] See, for example, R.B. Laughlin, Int. J. Mod. Phys. B 5, 1507 (1991).

[10] J.K. Jain, Phys. Rev. Lett. 63, 199 (1989).

[11] J.A. Simmons et al., Phys. Rev. Lett. 63, 1731 (1989); Phys. Rev. B 44, 12933 (1991); R.G. Clark al., Phys. Rev. Lett. 60, 1747 (1988); A.M. Chang and J.E. Cunningham, Surf. Sci. 229, 216 (1990).

[12] N. Byers and C.N. Yang, Phys. Rev. Lett. 7, 46 (1961).

[13] This actually implies that the fractionally charged quasiparticles must obey fractional statistics. See, S.A. Kivelson and M. Rocek, Phys. Lett. B 156, 85 (1989). 
[14] (a) S.A. Kivelson, Phys. Rev. Lett. 65, 3369 (1990); (b) D.J. Thouless, Phys. Rev. B 40, 12034 (1989); D.J. Thouless and Y. Gefen, Phys. Rev. Lett. 66, 806 (1991); Y. Gefen and D.J. Thouless, Phys. Rev. B 47, 10423 (1993); Pryadko and V.L. Pokrovsky, unpublished.

[15] P. Streda, S. Kucera, and A.H. MacDonald, Phys. Rev. Lett. 59, 1973 (1987); J.K. Jain and S.A. Kivelson, Phys. Rev. B 37, 4276 (1988).

[16] J.K. Jain and S.A. Kivelson, Phys. Rev. Lett. 60, 1542 (1988). Edge transport in the FQHE regime was demonstrated by J.K. Wang and V.J. Goldman, Phys. Rev. Lett. 67, 749 (1991).

[17] We will assume that the number of quasiparticles $K>>1$ so that order unity corrections can be neglected.

[18] J.K. Jain, Phys. Rev. B 41, 7653 (1990).

[19] For a more detailed discussion of the quasielectron statistics, see A. Karlhede, S.A. Kivelson, and S.L. Sondhi, in Prodeedings of the Ninth Jerusalem Winter School on Theoretical Physics, January 1992.

[20] S. Artz, T.H. Hansson, A. Karlhede, and T. Staab, Phys. Lett. B 267, 389 (1991).

[21] Also see, M. Ma and F.C. Zhang, Phys. Rev. Lett. 66, 1769 (1991).

[22] See, for example, P.A. Lee, Phys. Rev. Lett. 65, 2206 (1990). 


\section{Figure Caption:}

Figure 1. (a) Standard Aharonov Bohm geometry. (b) Schematic drawing of the proposed resonant tunneling experiment. The shaded area is the island of $\nu=p / q$ FQHE liquid surrounded by the $\nu^{\prime}=p^{\prime} / q^{\prime}$ FQHE liquid. The dashed lines show the most probable tunneling paths. 